\title{
Fault Detection of Train Center Plate Bolts Loss Using Modified LBP and Optimization Algorithm
}

\author{
Zhang Hongjian*, He Ping and Yang Xudong \\ Department of Control Science and Engineering, Harbin Institute of Technology, Harbin, Heilongjiang 150001, P.R. \\ China
}

\begin{abstract}
This paper presents a novel approach to fault detection of train center plate bolts loss based on Local Binary Patterns (LBP) and Gabor-GA optimization theory. A modified LBP operator including the positive-negative sign and magnitude components of local gray difference is introduced to extract much more texture information. Multi-channel Gabor wavelet with different scales and orientations is applied on the images to create new representations in the spatial domain. Then, the weight of each Gabor channel can be optimized through the Genetic Algorithm (GA) to obtain enhanced features. Finally, the weighted features are concatenated together and delivered into Support Vector Machine (SVM) network for classification. Experimental results show that the new approach can be an effective and reliable measure for monitoring fault.
\end{abstract}

Keywords: Fault detection, genetic algorithm, local binary patterns, support vector machine, train center plate bolts loss.

\section{INTRODUCTION}

Fault detection of train center plate bolts loss (TCPBL) is an important part of the trouble of moving freight car detection System (TFDS) which is highly demanded by the China Ministry of Railways today [1]. With trackside cameras taking photographs of the key parts of the running train, fault detection is accomplished automatically by computer image recognition, so the efficiency and reliability of train safety inspection are greatly improved [2-3]. TCPBL has a high incidence and can easily lead to the train derailment. In recent years, detection methods mainly focus on the statistical analysis of the shape and gray values of image for feature extraction, such as the constraint-rectangle calculator and gray average of local domain [4], the binary map and Hough transform [5] and the Haar-like features [6]. However these methods tend to suffer when variations in different factors (such as pose, illumination and occlusion etc.) are present. Therefore, the approach to increase the robustness of the system against different factors is researched.

The Local Binary Patterns (LBP) histogram is used to extract features. LBP is a simple yet efficient operator to describe the local texture information, which has achieved impressive results on face images [7-8]. A new personindependent facial expression recognition algorithm by Zhao et al. [9], whose feature vectors is the combination of the LBP features and the local statistic features of images after Gabor transform. Gabor wavelets have been widely used to extract the local frequency information from an image, which can estimate the strength of different frequency bands and orientations and give new representations in the spatial domain [10-13]. Gabor wavelets were applied on fabric images divided in windows to extract the texture features, and Principal Component Analysis (PCA) was used to reduce the dimension of feature vectors [14]. Furthermore, features of each Gabor channel have different contribution to the final classification. With features extracted from multichannel Gabor filters, a weighted voting face recognition method using the 40 different Gabor was reported by Nouyed et al. [15]. Gao et al. [16] pointed out that high recognition performance can be obtained by weighting the Local Gabor Magnitude Map (LGMM) based on the different contribution of each sub-pattern to the classification. Although the corresponding optimum weight of each Gabor channel is difficult to determine in theory, Genetic Algorithm (GA) is routinely used to generate useful solutions to parameter optimization problems [17]. With GA optimizing weights of a 38-dimensional feature set, further improvements in speaker recognition performance is attained [18]. Besides, Support Vector Machine (SVM) is a very powerful discriminative method for classification [19]. With features generated by concatenating the Gabor features of all image blocks, SVM can classify each fabric image as nondefective and defective [20].

Although an enormous amount of literature has been published on image recognition techniques, little research has been done on the research of TCPBL problem and it is still a challenging task to find a reliable detection method. In this paper, a useful approach to fault detection of TCPBL is proposed. A modified LBP operator is introduced to extract the feature of Gabor representations. Both the positivenegative sign and magnitude parts of local gray difference are coded; thus much more texture information is obtained. With weight optimized by GA, the features of each channel of Gabor representations are combined together and then classified by the SVM network. Experimental results indicate that the proposed method has much higher 
recognition rate compared with methods based on original LBP feature with no weight optimization.

The rest of this paper is organized as follows: Section 2 describes the algorithm of LBP feature extraction, Gabor-GA optimization, SVM classifier and the entire recognition method. Then the bolts region image databases and the experimental results are given in Section 3. Section 4 gives some conclusions and future plans.

\section{ALGORITHM DESCRIPTION}

\subsection{Modified LBP Operator}

The LBP operator is a powerful means to describe the local texture characteristics of images, which has good robustness on the influence of light illumination, angle and occlusion. The original LBP operator is defined as:

$\left.\mathrm{LBP}_{\mathrm{P}, \mathrm{R}}=\sum_{i=0}^{\mathrm{P}-1} \mathrm{~s}\left(\mathrm{~g}_{i}-\mathrm{g}_{c}\right) \cdot 2^{i}, \mathrm{~s} \mathrm{~g}_{i}-\mathrm{g}_{c}\right)=\left\{\begin{array}{cc}1 & \mathrm{~g}_{i} \geq \mathrm{g}_{c} \\ 0 & , \mathrm{~g}_{i}<\mathrm{g}_{c}\end{array}\right.$

where $\mathrm{g}_{c}$ is the gray value of the central pixel. $\mathrm{g}_{\mathrm{i}}(i=0, \ldots, \mathrm{P}-1)$ is $\mathrm{P}$ neighbor sampling points on a circle of radius R. According to Equation (1), LBP actually uses only the sign component during the local structure extraction. Apparently, this may lead to some incorrect matches. Two vectors shown in Fig. (1) have the same LBP vector. However, it is hard to say they have similar local structures.

\begin{tabular}{|l|l|l|}
\hline 163 & 21 & 124 \\
\hline 98 & 121 & 203 \\
\hline 188 & 119 & 67 \\
\hline
\end{tabular}

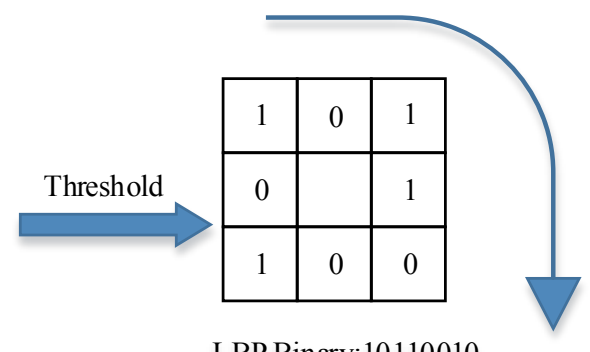

\begin{tabular}{|c|c|c|}
\hline 246 & 101 & 225 \\
\hline 16 & 121 & 157 \\
\hline 239 & 78 & 99 \\
\hline
\end{tabular}

LBP Binary:10110010

Fig. (1). Two structures with same LBP operator.

Although the sign component preserves most of local difference information, the magnitude component can also contribute additional discriminant information if it is properly used. Therefore, a modified LBP operator considering both the sign and magnitude components of the local difference is proposed. The sign component of the new operator is described as:

$s^{\prime}\left(g_{i}, g_{c}, t\right)=\left\{\begin{array}{l}1, g_{i} \geq g_{c}+t \\ 0,\left|g_{i}-g_{c}\right|<t \\ -1, g_{i} \leq g_{c}-t\end{array}\right.$

where $t$ is a user-defined threshold which can decrease the sensitivity to noise. For simplified calculation, the sign vector is divided into two parts: LBP_H operator ("- 1 " is coded as " 0 ") and LBP_L operator (" 1 " is coded as " 0 " and "-1" is coded as " 1 ").

The magnitude component of the new operator is defined as:

$\mathrm{LBP}_{-} M=\sum_{i=0}^{P-1} s\left(\left|g_{i}-g_{c}\right|, \mathrm{c}\right) \cdot 2^{P}, s(x, \mathrm{c})=\left\{\begin{array}{l}1, x \geq \mathrm{c} \\ 0, x<\mathrm{c}\end{array}\right.$

where $c$ is a threshold to be determined adaptively. Here we set it as the mean value of the neighborhood magnitude space. By coding both the sign and magnitude features into LBP codes and fusing them, much better texture classification results can be obtained than using only one of them. A typical modified LBP operator is shown in Fig. (2).

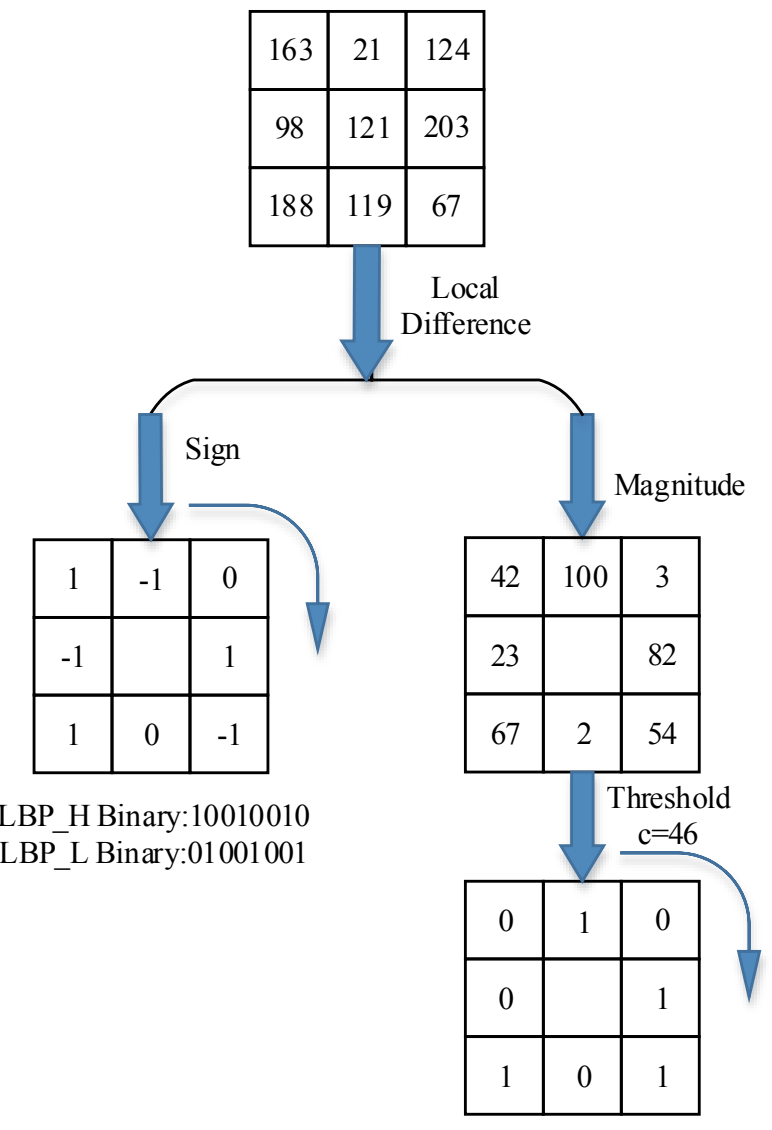

LBP_M Binary:01011010

Fig. (2). A modified LBP operator.

\subsection{Gabor-GA Optimization}

Gabor wavelet is a set of narrow band-pass filters with different scales and orientations which has a good resolution in the spatial domain. The Gabor wavelet transform of image is helpful to extract the features of image and also has well robustness to the local deformation, which is widely used in texture representation and target recognition. The kernel function of Gabor wavelets used in this paper is:

$G=\frac{u^{2}+v^{2}}{\delta^{2}} \cdot e^{-\frac{\left(u^{2}+v^{2}\right)\left(x^{2}+y^{2}\right)}{2 \delta^{2}}} \cdot\left[e^{-i(u x+v y)}-\mathrm{e}^{\frac{\delta^{2}}{2}}\right]$ 
where :

$k=\left(\begin{array}{l}u \\ v\end{array}\right)$

$u=\frac{k_{\max }}{f^{N u}} \cdot \cos \left(\frac{M u \cdot \pi}{\delta}\right)$

$v=\frac{k_{\max }}{f^{N u}} \cdot \sin \left(\frac{M u \cdot \pi}{\delta}\right)$

here $u$ and $v$ are the orientation and scale of the gabor kernel and $f=\sqrt{2}, \delta=2 \pi$ are selected.

Given a gray-level image, which has been preprocessed already, we take the convolution of it with a Gabor kernel for image representation. In this paper, 40 Gabor wavelet kernels are used at five scales and eight orientations, thus $N u=\{0,1, \cdots, 4\} \quad$ and $M u=\{0,1, \cdots, 7\} \quad$. The new representations is obtained by convolving the input image with these 40 Gabor kernels. Then, the output images of 40 Gabor channels are acquired, which represent the information of original image in different frequency scales and texture directions. Finally, the features of each Gabor channel are extracted and combined together. These features of different Gabor channel are usually treated equally or selected partially in the traditional methods, however the features of every channel distinctively contribute to the recognition task. It will have a positive influence on the recognition task to weight each Gabor channel differently. Although the contribution of each channel is invariant, it lacks of theory to calculate the corresponding global optimum weights.

Fig. (3) explains the schematic of the Gabor-GA optimization algorithm that enhance the final features by optimally adjusting the feature weight of each Gabor channel. Apparently, the design variables are elements of the weight for each Gabor channel, where GA is used to seek for optimal weights of each Gabor channel with its powerful capability in global-optimization. Thus, the final feature is acquired by concatenating the weighted features of 40 Gabor channel together.

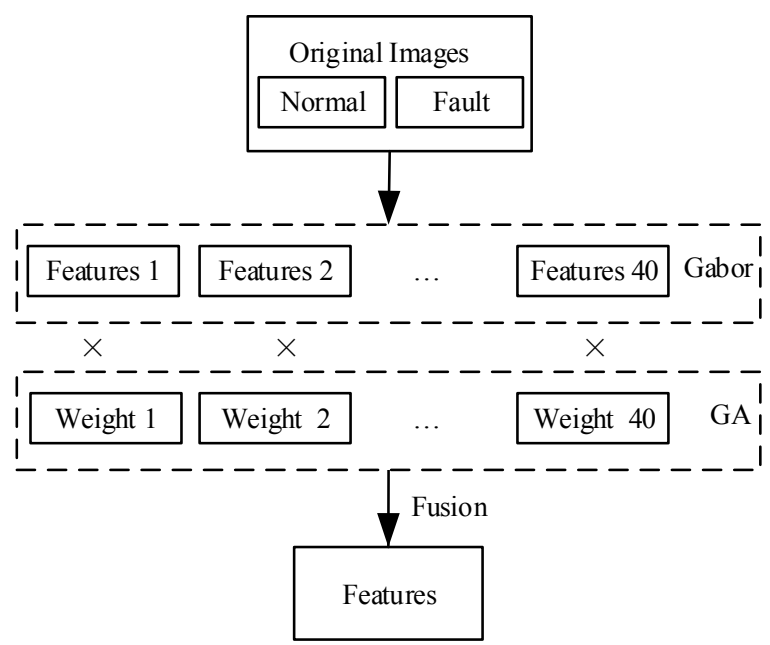

Fig. (3). A schematic block diagram for the process of Gabor-GA.

\subsection{SVM Classifier}

Support Vector Machines (SVM) is a machine-learning tool based on the statistical learning theory and the structure risk minimization construct. It is a good solution to problems such as small sample, high dimension, over learning and local minimum. In this work, RBF kernel shown in Equation (6) is selected, which shows good learning performance in the practical application.

$\mathrm{K}\left(\mathrm{x}_{i}, \mathrm{x}_{j}\right)=\exp \left\{-\frac{\left|\mathrm{x}_{i}-\mathrm{x}_{j}\right|^{2}}{2 \sigma^{2}}\right\}$

However it is difficult to determine the appropriate values of the penalty parameter $c$ and the kernel parameter $\sigma$. The best combination of $c$ and $\sigma$ is often selected by a grid search with exponentially growing sequences which is the defect in the practical application. In this paper, the parameter selection is optimized by GA. Within the parameter groups of $c$ and $\sigma$ with the highest fitness, the one with the smallest $c$ is chosen as the best parameter combination.

\subsection{Fault Detection Method}

The entire fault detection method is shown in Fig. (4).

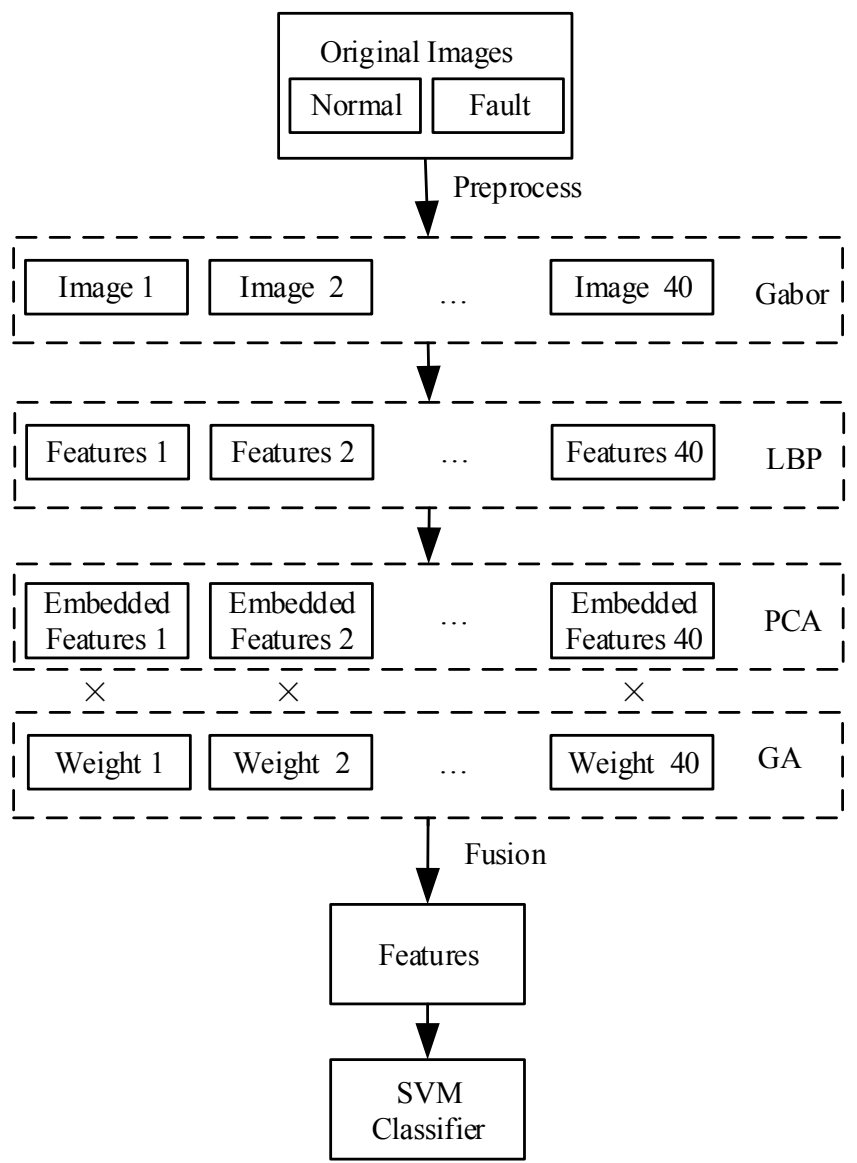

Fig (4). The framework of the whole system.

Gabor wavelet transform is the optimal image representation from different scales and orientations. However the feature dimensionality of the Gabor representation is 40 
times of the original image which contains massive redundant information and will influence the recognition efficiency. Therefore, the modified LBP operator is used to extract the features of each Gabor channel. Considering 40 Gabor channels, the whole LBP histograms still contain a large amount of features. Consequently, PCA is adopted on each Gabor channel to reduce the dimensionality, which can retain most of the important information of the original histograms and save computation time for classification. With GA optimizing the corresponding weight of each channel, the final feature are fusion of all the 40 channels. Finally, the fault detection is completed by the SVM classifier.

\section{EXPERIMENTS, RESULTS AND DISCUSSIONS}

\subsection{Experiment Database}

The images are shot in the process of train movement with cameras exposed outside. Different environment such as weather and illumination have a significant impact on the brightness and contrast of the acquired images. It might have the problems of blur, poor illumination, excess exposure and occlusion. Fig. (5) is a typical field image photographed by high speed trackside camera. There should be four fixed bolts normally, however one bolt has been lost while the upper of another bolt is covered by the drawbar.

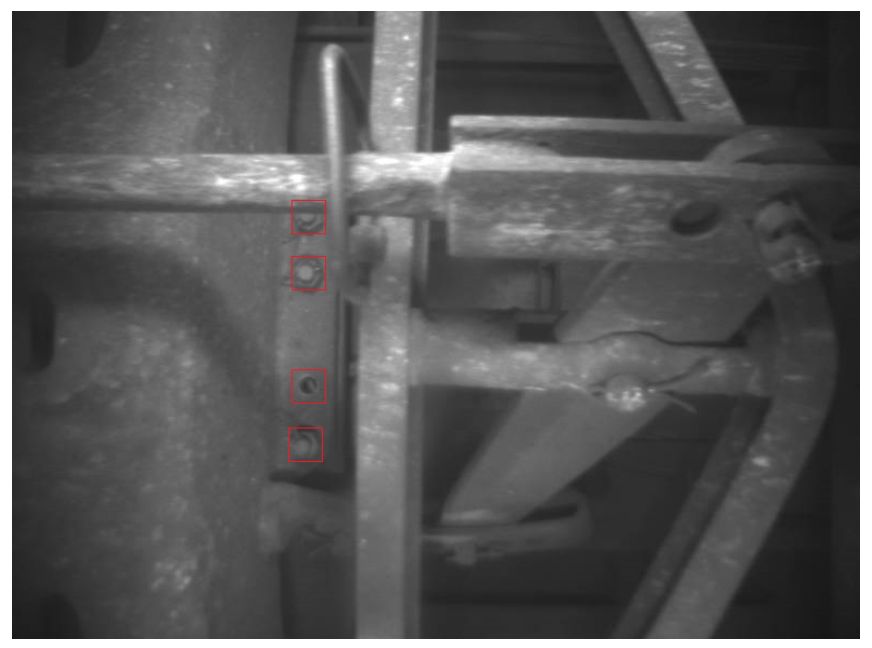

Fig. (5). A typical field image.

This paper mainly studies on the automatic recognition method of bolts region images after segmentation of region of interest (ROI). Therefore, the experiment database is from the segmentation of ROI of the field images with fault detection manually checked, which contains 500 normal images and 500 fault images. Each image has a resolution of $32 \times 32$ pixels. Some of them are illustrated in Fig. (6).

\subsection{Experiment Results and Discussions}

The main steps of the proposed approach are as described below:

1. The image preprocess procedure including Gamma correction, DOG filter and contrast normalization is performed and the preprocessed results are shown in Fig. (7).

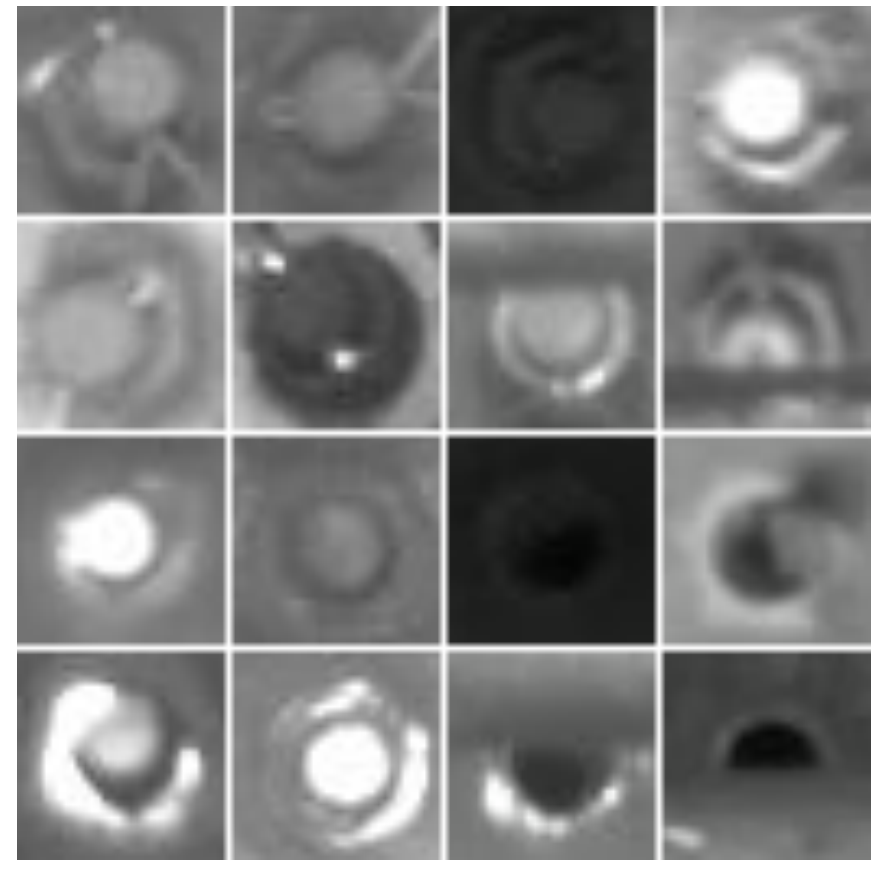

Fig. (6). Some samples of bolts region images database.

2. The preprocessed images are filtered by multichannel Gabor wavelets with five scales and eight orientations. Then the modified LBP histograms of each Gabor channel are extracted.

3. PCA is used on each channel. The classification accuracy varies with the number of bins of LBP histograms maintained during PCA process. The contrast results of different number of bins maintained of LBP and modified LBP features of each channel are shown in Fig. (8); thus the best number of bins maintained is selected as 8 .

4. The Gabor-GA optimization algorithm is used to obtain the best weights of 40 Gabor channels. The best and average fitness (i.e. cross validation rate) of the chromosome populations during the GA evolution are shown in Fig. (9).

5. The weighted feature vectors of every channel are serial connected and then delivered into the SVM network for the final classification.

To save the computation time for bins number in Step 3 and the weight optimization in Step 4, the 10-fold Cross Validation is adopted. In Step 5, the Leave-One-Out Cross Validation is adopted to reduce the influence of stochastic factors and improve the reliability of classification accuracy.

To evaluate the performance of this approach, several different feature extraction methods with the Nearest Neighbor (NN) classifier and the SVM classifier are compared and their recognition results are given in Table $\mathbf{1 .}$

In Table 1, some abbreviations are expressed as follows:

PP --- the preprocess procedure;

MLBP --- the proposed modified LBP operator;

The chi-square distance $\chi^{2}$ shown in Equation (7) is selected as the dissimilarity measurement of $\mathrm{NN}$ classifier: 
The test sample is assigned to the class of model that minimizes $\chi^{2}$.

$$
\chi^{2}(\mathrm{~T}, \mathrm{M})=\sum_{i} \frac{\left(T_{i}-M_{\mathrm{i}}\right)^{2}}{T_{i}+M_{i}}
$$

where,

$T_{i}$--- the value of the sample $T$ at $i^{\text {th }}$ bin;

$M_{i}$--- the value of the model image $M$ at $i^{\text {th }}$ bin;

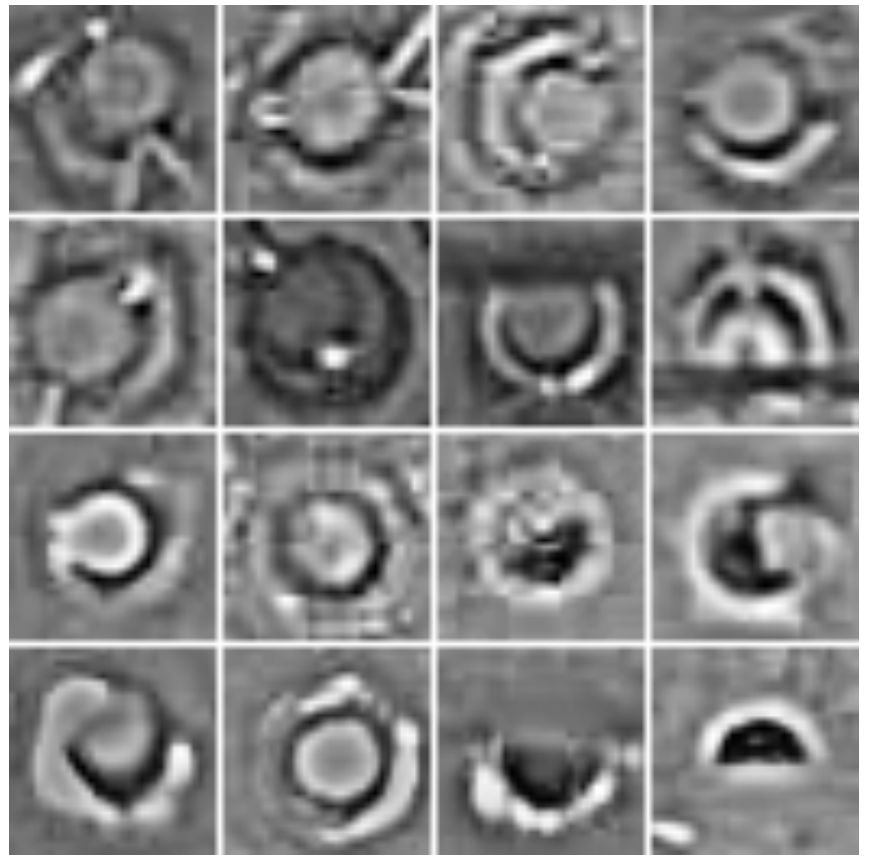

Fig. (7). The preprocessed images.

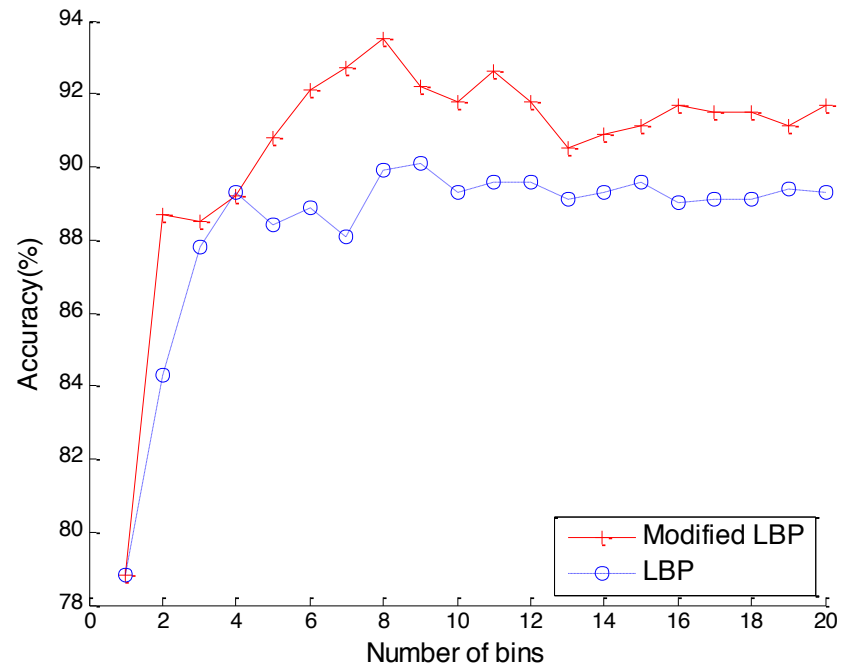

Fig. (8). Accuracy of different number of bins maintained.

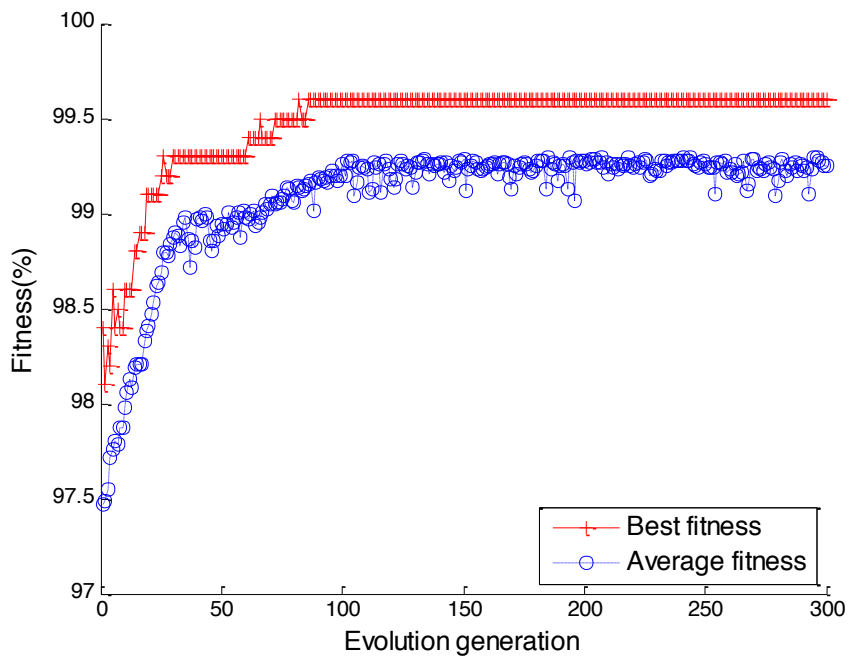

Fig. (9). Fitness during the evolution.

The results show that the preprocess method is useful to reduce the influence of shooting limitation. The modified LBP operator always has much higher accuracy than the basic one because it can maintain much more local difference information. Furthermore, the Gabor-GA optimization algorithm can significantly increase the recognition rate. The combination of Gabor filter and PCA can effectively extract abundant texture features of new representations with different scales and orientations in a lower dimensionality. Moreover, the weight of each channel is optimized by GA which can also enhance the feature and then improve the recognition performance. Besides, SVM classifier always has much higher recognition rate than $\mathrm{NN}$, which shows its powerful capability in fault detection.

Table 1. Performance comparisons of different methods.

\begin{tabular}{|c|c|c|}
\hline Feature Extraction Method & NN (\%) & SVM (\%) \\
\hline \hline LBP & 60.7 & 91.5 \\
\hline MLBP & 64.9 & 93.7 \\
\hline PP +LBP & 66.2 & 94.3 \\
\hline PP +MLBP & 67.4 & 95.3 \\
\hline PP +LBP+PCA & 67.3 & 95.2 \\
\hline PP +MLBP+PCA & 72.4 & 97.6 \\
\hline PP +Gabor+LBP+PCA & 90.1 & 97.7 \\
\hline PP +Gabor+MLBP+PCA & 93.5 & 98.2 \\
\hline PP +LBP+PCA+Gabor-GA Optimization & 91.3 & 98.6 \\
\hline PP +MLBP+PCA+Gabor-GA Optimization & 93.7 & 99.1 \\
\hline
\end{tabular}

\section{CONCLUSION}

The automatic fault detection of TCPBL is a new application problem in pattern recognition and a novel 
approach is given in this paper. This approach has two major features: (1) a modified LBP operator is presented to extract sign and magnitude feature vector of local difference, which can obtain much more discrimination information; (2) Gabor-GA provides a helpful solution to get optimized features by the weight fusion of different channel. The proposed approach is excellent, proved by using field images database and compared with other methods. Future research should be focused on reducing its computational time and applying it on other TFDS fault detection problems.

\section{CONFLICT OF INTEREST}

The author confirms that this article content has no conflict of interest.

\section{ACKNOWLEDGEMENTS}

Declared none.

\section{REFERENCES}

[1] Z.X. Zhu, and G.Y. Wang, "A fast potential fault regions locating method used in inspecting freight cars", Journal of Computers, vol. 9, pp. 1266-1273, 2012

[2] K. Zhou, M. Lu, G. Wang and B.Y. Ren, "Monitoring data transmission technology in 5T system based on message", China Railway Science, vol. 29, pp. 113-118, 2008.

[3] Z.H. Liu, D.Y. Xiao and Y.M. Chen, "Displacement fault detection of bearing weight saddle in TFDS based on Hough transform and symmetry validation", In: Proceedings of the $9^{\text {th }}$ International Conference on Fuzzy Systems and Knowledge Discovery, Chongqing, China, 2012, pp. 1404-1408.

[4] P. Dai, X.D. Yang, P. He and H.J Zhang, "Automatic recognition for losing of train bogie center plate screw based on multiple-fuzzy relation tree," In: Proceedings of the International Workshop on Intelligent Systems and Applications, Wuhan, China, 2009, pp. 1-5.

[5] X.D. Yang, L.J. Ye and J.B. Yuan, "Research of computer vision fault recognition algorithm of center plate bolts of train", In: Proceedings of the International Conference on Instrumentation, Measurement, Computer, Communication and Control, Beijing, China, 2011, pp. 978-981

[6] N. Li, Z.Z. Wei, Z.P. Cao and X.G. Wei, "Automatic fault recognition for losing of train bogie center plate bolt", In: Proceedings of the $14^{\text {th }}$ International Conference on Communication Technology, Chengdu, China, pp. 1001-1005, 2012.

[7] T. Ahonen, A. Hadid and M. Pietikäinen, "Face description with local binary patterns: Application to face recognition", IEEE
Transactions on Pattern Analysis and Machine Intelligence, vol. 28, pp. 2037-2041, 2006

[8] Z.H. Guo, L. Zhang and D. Zhang, "A completed modeling of local binary pattern operator for texture classification", IEEE Transactions on Image Processing, vol. 19, pp. 1657-1663, 2010.

[9] Q.Y. Zhao, B.C. Pan, J.J. Pan and Y.Y. Tang, "Facial expression recognition based on fusion of Gabor and LBP features", In: Proceedings of the International Conference on Wavelet Analysis and Pattern Recognition, Hong Kong, China, 2008, pp. 362-367.

[10] L. Shen, L. Bai and M. Fairhurst, "Gabor wavelets and general discriminant analysis for face identification and verification", Image and Vision Computing, vol. 25, pp. 553-563, 2007.

[11] S. Wang, Y. Xia, Q. Liu, J. Luo, Y. Zhu and D.D. Feng, "Gabor feature based nonlocal means filter for textured image denoising", Journal of Visual Communication and Image Representation, vol. 23, pp. 1008-1018, 2012

[12] L. Bissi, G. Baruffa, P. Placidi, E. Ricci, A. Scorzoni and P. Valigi, "Patch based yarn defect detection using Gabor filters", In: Proceedings of the $9^{\text {th }}$ IEEE International Instrumentation and Measurement Technology Conference, Graz, Austria, 2012, pp. 240-244.

[13] L.B. Céu and A.T. Mário, "Defect detection in textile images using Gabor filters", Lecture Notes in Computer Science, vol. 3212, pp. 841-848, 2004

[14] A. Basturk, H. Ketencioglu, Z. Yugnak and M.E. Yuksel, "Inspection of defects in fabrics using Gabor wavelets and principle component analysis", In: Proceedings of the $9^{\text {th }}$ International Symposium on Signal Processing and Its Applications, Sharjah, United Arab Emirates, 2007, pp. 1-4.

[15] I. Nouyed, M.A. Amin, B. Poon and H. Yan, "Human face recognition using weighted vote of Gabor magnitude filters", In: Proceedings of the $7^{\text {th }}$ International Conference on Information Technology and Application, Sydney, Australia, 2011, pp. 36-40.

[16] T. Gao, X. Ma and J.G. Wang, "Particle occlusion face recognition using adaptively weighted local Gabor filters", Journal of Computational Information Systems, vol. 8, pp.5271-5277, 2012.

[17] O. David, N.S. Netanyahu, and Y. Rosenberg, "Genetic algorithms for automatic object movement classification", Lecture Notes in Computer Science, vol. 6935, 2011, pp. 258-265.

[18] M. Zamalloa, G. Bordel, L.J. Rodríguez M. Penagarikano, and J. Uribe, "Using genetic algorithms to weight acoustic features for speaker recognition", In: Proceedings of the Annual Conference of the International Speech Communication Association, Pittsburgh, PA, USA, 2006, pp. 909-912.

[19] Y.T. Tsai and J.T. Hwang, "Texture analysis using Gabor filters for image classification based on SVM", In: Proceedings of the $32^{\text {nd }}$ Asian Conference on Remote Sensing, Tapei, Taiwan, 2011, pp. 2107-2112

[20] S.H. Ding, C.L. Li and X.F. Liu, "Fabric defect detection scheme based on Gabor filter and PCA", Advanced Materials Research, vol. 482, pp.159-163, 2012.

(C) Hongjian et al.; Licensee Bentham Open.

This is an open access article licensed under the terms of the (https://creativecommons.org/licenses/by/4.0/legalcode), which permits unrestricted, non-commercial use, distribution and reproduction in any medium, provided the work is properly cited. 